\title{
Kinetic Analysis of Discrete Path Sampling Stationary Point Databases
}

\author{
Semen A. Trygubenko* and David J. Wales ${ }^{\dagger}$ \\ University Chemical Laboratories, Lensfield Road, \\ Cambridge CB2 1EW, UK
}

September 21, 2018

\begin{abstract}
Analysing stationary point databases to extract phenomenological rate constants can become time-consuming for systems with large potential energy barriers. In the present contribution we analyse several different approaches to this problem. First, we show how the original rate constant prescription within the discrete path sampling approach can be rewritten in terms of committor probabilities. Two alternative formulations are then derived in which the steady-state assumption for intervening minima is removed, providing both a more accurate kinetic analysis, and a measure of whether a two-state description is appropriate. The first approach involves running additional short kinetic Monte Carlo (KMC) trajectories, which are used to calculate waiting times. Here we introduce 'leapfrog' moves to second-neighbour minima, which prevent the KMC trajectory oscillating between structures separated by low barriers. In the second approach we successively remove minima from the intervening set, renormalising

\footnotetext{
*E-mail: sat39@cam.ac.uk

${ }^{\dagger}$ E-mail: dw34@cam.ac.uk
} 
the branching probabilities and waiting times to preserve the mean first-passage

times of interest. Regrouping the local minima appropriately is also shown to speed up the kinetic analysis dramatically at low temperatures. Applications are described where rates are extracted for databases containing tens of thousands of stationary points, with effective barriers that are several hundred times $k_{B} T$.

\section{Introduction}

A great deal of effort is currently focused on the development of new methods to treat 'rare events'. A number of these methods employ a coarse-graining approximation of some kind to the phase space. ${ }^{1-7}$ Examples include the interface formulation ${ }^{1}$ of transition path sampling, ${ }^{2,3}$ Markovian state models, ${ }^{4}$ milestoning, ${ }^{5}$ master equation approaches, ${ }^{6,8}$ and discretized reaction paths. ${ }^{7}$ The present contribution focuses on the discrete path sampling (DPS) approach, ${ }^{9-11}$ which produces a database of stationary points from the underlying potential energy surface (PES). Several new methods are developed for extracting phenomenological two-state rate constants from this database. As well as speeding up the kinetic analysis, we can also determine the extent to which a two-state description is appropriate.

The particular coarse-graining approach considered here focuses on a formally exact partitioning of the PES into the basins of attraction? of all the local minima. ${ }^{10}$ The corresponding superposition approach to thermodynamics is based upon the same division of the partition function. ${ }^{10,12}$ Kunz and Berry ${ }^{13}$ extended this scheme to treat kinetics by including transition states, which are here defined geometrically, as stationary points that possess a single negative Hessian eigenvalue. ${ }^{14}$ They employed statistical rate theory to calculate individual minimum-to-minimum rate constants, and a master equation approach ${ }^{15,16}$ to extract global dynamics. Unfortunately, the number of stationary points is generally expected to scale exponentially with system size, ${ }^{12,17}$ and we must therefore derive an appropriate sampling scheme in order to represent the kinetics 
properly. The DPS approach was introduced specifically to deal with this problem. ${ }^{9,10}$

To start a DPS analysis we must have an order parameter that enables us to identify local minima, $\mathrm{a} \in \mathrm{A}$ and $\mathrm{b} \in \mathrm{B}$, which belong to the two end-point states of interest, $\mathrm{A}$ and B. Minima that do not belong to either set will be labelled $i \in I$, where 'I' stands for 'intervening'. Local equilibrium must be established within each of the A and $\mathrm{B}$ regions relative to their interconversion rate for two-state kinetics to apply. The original DPS formulation then showed how phenomenological two-state rate constants $k_{\mathrm{AB}}$ and $k_{\mathrm{BA}}$ could be written as a sum over discrete paths between $\mathrm{A}$ and $\mathrm{B}$, so long as minima in the I set could be treated according to the steady-state approximation. ${ }^{9}$ Here a discrete path is defined as a sequence of local minima and the transition states that connect them. Starting from an initial discrete path that connects A and B, the DPS procedure generates new discrete paths by a directed search for additional transition states and local minima that can be merged with existing paths. For simple cases, where only a few discrete paths make a significant contribution, $k_{\mathrm{AB}}$ and $k_{\mathrm{BA}}$ are obtained from summing the corresponding terms. ${ }^{9}$ However, for more complicated pathways there may be many terms that contribute. In particular, the databases of stationary points that are constructed during the search for new paths generally contain vastly more $\mathrm{A} \leftrightarrow \mathrm{B}$ discrete paths than are explicitly considered during the sampling procedure. However, all these contributions can be summed using a matrix multiplication approach based upon results from graph theory. ${ }^{9}$ In fact these sums are equivalent to the committor probabilities discussed in $₫ 2$, and it is more efficient to calculate them using the successive overrelaxation technique. ${ }^{18}$

Overall rates can also be extracted from DPS stationary point databases using master equation ${ }^{15}$ and kinetic Monte Carlo ${ }^{19-21}$ (KMC) methods. ${ }^{9}$ These approaches do not require the steady-state approximation for minima in the I set, and hence they enable us to check whether this approximation is valid. Unfortunately, numerical problems often arise in the master equation approach, ${ }^{9}$ although grouping and pruning the sta- 
tionary point database may help to circumvent this issue. ${ }^{10,22} \mathrm{KMC}$ calculations do not experience the same difficulties, but can become very slow if the potential energy barrier between the $\mathrm{A}$ and $\mathrm{B}$ regions is large compared to $k_{B} T .{ }^{23,24}$ Importance sampling KMC schemes have recently been suggested to overcome these problems, ${ }^{23,24}$ but we have not succeeded in applying them to databases of the complexity considered in the present work. Instead we have developed the combined committor probability/KMC and graph transformation schemes described in the following sections.

\section{Two-State Rate Constant Expressions}

We start from a linear master equation formulation of the kinetics, ${ }^{15,16}$ which immediately invokes the assumption of Markovian dynamics:

$$
\begin{aligned}
& \frac{d P_{\mathrm{a}}(t)}{d t}=\sum_{\alpha} k_{\mathrm{a} \alpha} P_{\alpha}(t)-P_{\mathrm{a}}(t) \sum_{\alpha} k_{\alpha \mathrm{a}}, \\
& \frac{d P_{\mathrm{b}}(t)}{d t}=\sum_{\alpha} k_{\mathrm{b} \alpha} P_{\alpha}(t)-P_{\mathrm{b}}(t) \sum_{\alpha} k_{\alpha \mathrm{b}}, \\
& \frac{d P_{\mathrm{i}}(t)}{d t}=\sum_{\alpha} k_{\mathrm{i} \alpha} P_{\alpha}(t)-P_{\mathrm{i}}(t) \sum_{\alpha} k_{\alpha \mathrm{i}},
\end{aligned}
$$

where $P_{\alpha}(t)$ is the occupation probability of minimum $\alpha$ at time $t$ and $k_{\alpha \beta}$ is the rate constant for $\alpha \leftarrow \beta$ transitions between minima $\alpha$ and $\beta$, which are directly connected by a transition state on the PES. We assume that minima within the A and B sets are in local mutual equilibrium, so that

$$
P_{\mathrm{a}}(t)=\frac{P_{\mathrm{a}}^{\mathrm{eq}} P_{\mathrm{A}}(t)}{P_{\mathrm{A}}^{\mathrm{eq}}} \quad \text { and } \quad P_{\mathrm{b}}(t)=\frac{P_{\mathrm{b}}^{\mathrm{eq}} P_{\mathrm{B}}(t)}{P_{\mathrm{B}}^{\mathrm{eq}}},
$$

where $P_{\mathrm{A}}(t)=\sum_{\mathrm{a} \in \mathrm{A}} P_{\mathrm{a}}(t)$, etc., and the superscript 'eq' stands for 'equilibrium'. In the original derivation of two-state phenomenological rate constants within the DPS approach we also applied the steady-state approximation for each intervening minimum, i. These approximations enable us to replace the probabilities $P_{\mathrm{i}}(t)$ in an iterative fashion to obtain

$$
k_{\mathrm{AB}}^{\mathrm{SS}}=\frac{1}{P_{\mathrm{B}}^{\mathrm{eq}}} \sum_{\mathrm{A} \leftarrow \mathrm{B}}^{\prime} \frac{k_{\mathrm{ai}_{1}} k_{\mathrm{i}_{1} \mathrm{i}_{2}} \cdots k_{\mathrm{i}_{n-1} \mathrm{i}_{n}} k_{\mathrm{i}_{n} \mathrm{~b}} P_{\mathrm{b}}^{\mathrm{eq}}}{\sum_{\alpha_{1}} k_{\alpha_{1} \mathrm{i}_{1}} \sum_{\alpha_{2}} k_{\alpha_{2} \mathrm{i}_{2}} \cdots \sum_{\alpha_{n}} k_{\alpha_{n} \mathrm{i}_{n}}},
$$


and an analogous expression for $k_{\mathrm{BA}}^{\mathrm{SS}}$. Here the sum is over all discrete paths that begin at any minimum $\mathrm{b} \in \mathrm{B}$ and end at any minimum $\mathrm{a} \in \mathrm{A}$, and the prime denotes a restriction that only intervening minima $\mathrm{i} \in \mathrm{I}$ can be revisited. The 'SS' superscript is introduced to distinguish this result, which includes the steady-state approximation for minima in the I set. Each factor of the form $k_{\mathrm{ji}} / \sum_{\alpha} k_{\alpha \mathrm{i}}$ can be written in terms of the corresponding branching probability, $P_{\mathrm{ji}}=k_{\mathrm{ji}} / \sum_{\gamma} k_{\gamma \mathrm{i}}$, where the sum in the denominator runs over all minima $\gamma$ directly connected to $i$ :

$$
\begin{aligned}
k_{\mathrm{AB}}^{\mathrm{SS}} & =\frac{1}{P_{\mathrm{B}}^{\mathrm{eq}}} \sum_{\mathrm{A} \leftarrow \mathrm{B}}^{\prime} P_{\mathrm{ai}_{1}} P_{\mathrm{i}_{1} \mathrm{i}_{2}} \cdots P_{\mathrm{i}_{n-1} \mathrm{i}_{n}} k_{\mathrm{i}_{n} \mathrm{~b}} P_{\mathrm{b}}^{\mathrm{eq}} \\
& =\frac{1}{P_{\mathrm{B}}^{\mathrm{eq}}} \sum_{\mathrm{A} \leftarrow \mathrm{B}}^{\prime} P_{\mathrm{ai}_{1}} P_{\mathrm{i}_{1} \mathrm{i}_{2}} \cdots P_{\mathrm{i}_{n-1} \mathrm{i}_{n}} P_{\mathrm{i}_{n} \mathrm{~b}} \tau_{\mathrm{b}}^{-1} P_{\mathrm{b}}^{\mathrm{eq}}
\end{aligned}
$$

where $\tau_{\mathrm{b}}=1 / \sum_{\alpha} k_{\alpha \mathrm{b}}$ is the mean waiting time for escape from minimum b to any of its neighbouring minima that are linked to it by a single transition state. This expression can be written in a much simpler form by recognising that the sum of the products of branching probabilities over all paths from minimum b to minima in the A region that revisit only I minima is the committor probability, $C_{\mathrm{b}}^{\mathrm{A}}$ :

$$
C_{\mathrm{b}}^{\mathrm{A}}=\sum_{\mathrm{A} \leftarrow \mathrm{b}}^{\prime} P_{\mathrm{ai}_{1}} P_{\mathrm{i}_{1} \mathrm{i}_{2}} \cdots P_{\mathrm{i}_{n-1} \mathrm{i}_{n}} P_{\mathrm{i}_{n} \mathrm{~b}}
$$

so that

$$
k_{\mathrm{AB}}^{\mathrm{SS}}=\frac{1}{P_{\mathrm{B}}^{\mathrm{eq}}} \sum_{\mathrm{b} \in \mathrm{B}} \frac{C_{\mathrm{b}}^{\mathrm{A}} P_{\mathrm{b}}^{\mathrm{eq}}}{\tau_{\mathrm{b}}}, \quad \text { and } \quad k_{\mathrm{BA}}^{\mathrm{SS}}=\frac{1}{P_{\mathrm{A}}^{\mathrm{eq}}} \sum_{\mathrm{a} \in \mathrm{A}} \frac{C_{\mathrm{a}}^{\mathrm{B}} P_{\mathrm{a}}^{\mathrm{eq}}}{\tau_{\mathrm{a}}} .
$$

For example, $C_{\mathrm{b}}^{\mathrm{A}}$ is the probability that a stochastic trajectory started from minimum $\mathrm{b}$ will encounter an $\mathrm{A}$ minimum before a $\mathrm{B}$ minimum, while $C_{\mathrm{b}}^{\mathrm{B}}=1-C_{\mathrm{b}}^{\mathrm{A}}$ is the probability that a trajectory will encounter the B region before A. The parameter $P_{\alpha}^{\text {fold }}$, defined as the probability that a protein will fold before unfolding, starting from some initial condition $\alpha,{ }^{4,25,26}$ is a more specific example of a committor probability.

To derive corresponding expressions without invoking the steady-state approximation for minima in the I set we write the master equation in terms of transitions between members of the $\mathrm{A}$ and $\mathrm{B}$ sets and the corresponding effective rate constants $K_{\mathrm{ab}}$ and 
$K_{\text {ba }}:$

$$
\begin{aligned}
k_{\mathrm{AB}}^{\mathrm{NSS}} & =\frac{1}{P_{\mathrm{B}}^{\mathrm{eq}}} \sum_{\mathrm{b} \in \mathrm{B}} \sum_{\mathrm{a} \in \mathrm{A}} K_{\mathrm{ab}} P_{\mathrm{b}}^{\mathrm{eq}}=\frac{1}{P_{\mathrm{B}}^{\mathrm{eq}}} \sum_{\mathrm{b} \in \mathrm{B}} K_{\mathrm{Ab}} P_{\mathrm{b}}^{\mathrm{eq}}, \\
\text { and } \quad k_{\mathrm{BA}}^{\mathrm{NSS}} & =\frac{1}{P_{\mathrm{A}}^{\mathrm{eq}}} \sum_{\mathrm{a} \in \mathrm{A}} \sum_{\mathrm{b} \in \mathrm{B}} K_{\mathrm{ba}} P_{\mathrm{a}}^{\mathrm{eq}}=\frac{1}{P_{\mathrm{A}}^{\mathrm{eq}}} \sum_{\mathrm{a} \in \mathrm{A}} K_{\mathrm{Ba}} P_{\mathrm{a}}^{\mathrm{eq}},
\end{aligned}
$$

where the superscript 'NSS' stands for 'non-steady-state'. $K_{\mathrm{Ab}}$ is the effective rate constant for transitions from minimum b to the A minima, and $K_{\mathrm{Ba}}$ is the corresponding rate constant for transitions from minimum a to the $\mathrm{B}$ minima; the minima in question will not generally be connected by a single transition state. Treating transitions from minimum $\mathrm{b}$ to the $\mathrm{A}$ and $\mathrm{B}$ regions as independent Poisson processes with the above rate constants yields a mean waiting time between transitions of $t_{\mathrm{b}}=1 /\left(K_{\mathrm{Ab}}+K_{\mathrm{Bb}}\right)$. Here $K_{\mathrm{Bb}}$ corresponds to the effective rate constant for a trajectory to return to any member of the $\mathrm{B}$ set starting from b. However, $K_{\mathrm{Ab}} /\left(K_{\mathrm{Ab}}+K_{\mathrm{Bb}}\right)=K_{\mathrm{Ab}} t_{\mathrm{b}}$ can be identified with the committor probability $C_{\mathrm{b}}^{\mathrm{A}}$, and hence we obtain

$$
k_{\mathrm{AB}}^{\mathrm{NSS}}=\frac{1}{P_{\mathrm{B}}^{\mathrm{eq}}} \sum_{\mathrm{b} \in \mathrm{B}} \frac{C_{\mathrm{b}}^{\mathrm{A}} P_{\mathrm{b}}^{\mathrm{eq}}}{t_{\mathrm{b}}}, \quad \text { and } \quad k_{\mathrm{BA}}^{\mathrm{NSS}}=\frac{1}{P_{\mathrm{A}}^{\mathrm{eq}}} \sum_{\mathrm{a} \in \mathrm{A}} \frac{C_{\mathrm{a}}^{\mathrm{B}} P_{\mathrm{a}}^{\mathrm{eq}}}{t_{\mathrm{a}}} .
$$

The only difference between the expressions in equations (6) and (8) is that the average waiting times between events are interpreted differently. In (8) $t_{\mathrm{b}}$ is the mean waiting time between events corresponding to transitions between $\mathrm{b}$ and any minima in $\mathrm{A} \cup \mathrm{B}$, including return to $\mathrm{b}$ itself. However, in (6) $\tau_{\mathrm{b}}$ is simply the mean waiting time for a transition from minimum b to a minimum connected to it by a single transition state. Clearly $\tau_{\mathrm{b}} \leq t_{\mathrm{b}}$. Furthermore, in the steady-state limit for the intervening minima, the corresponding waiting times $\tau_{\mathrm{i}}$ must be negligible, so that $t_{\mathrm{b}} \rightarrow \tau_{\mathrm{b}}$, and $k_{\mathrm{AB}}^{\mathrm{NSS}} \rightarrow k_{\mathrm{AB}}^{\mathrm{SS}}$, as expected.

To determine the committor probabilities $C_{\mathrm{b}}^{\mathrm{A}}$ we use the relation (a first-step anal$\left.\operatorname{ysis}^{27}\right)$

$$
C_{\alpha}^{\mathrm{A}}=\sum_{\beta} C_{\beta}^{\mathrm{A}} P_{\beta \alpha}
$$

which is analogous to the expression used for $P_{\alpha}^{\text {fold }}$ in reference [4]. When calculating $k_{\mathrm{BA}}^{\mathrm{SS}}$ and $k_{\mathrm{BA}}^{\mathrm{NSS}}$ we simply replace the 'A' superscripts by 'B' in equation (9). The sum 
over $\beta$ includes all minima directly connected to minimum $\alpha$ by a single transition

state, as these are the only non-zero branching probabilities. The $C_{\mathrm{b}}^{\mathrm{A}}$ were calculated iteratively using the successive overrelaxation technique with an extrapolation factor of $1.999 .^{18}$ The sparse matrix whose elements are the branching probabilities $P_{\beta \alpha}$ was represented using the compressed row storage scheme. ${ }^{28}$

As in previous DPS calculations we can use harmonic densities of states to calculate the equilibrium occupation probabilities, along with expressions from statistical rate theory $^{29}$ for the individual rate constants, $k_{\alpha \beta}$, corresponding to transitions between directly connected minima. Once the committor probabilities have been calculated we have all the quantities required to evaluate $k_{\mathrm{AB}}^{\mathrm{SS}}$ and $k_{\mathrm{BA}}^{\mathrm{SS}}$. To calculate the mean waiting time $t_{\mathrm{b}}$ for transitions from $\mathrm{b} \in \mathrm{B}$ to any $\mathrm{A}$ or $\mathrm{B}$ minimum, including revisits to I minima, we employ the kinetic Monte Carlo (KMC) approach. ${ }^{19-21}$ Stochastic $^{2}$ trajectories are simply run from minimum b until we reach any minimum belonging to the $\mathrm{A}$ or $\mathrm{B}$ sets, and $t_{\mathrm{b}}$ is evaluated as an average over a number of independent trials.

The above formulation in terms of the waiting times $t_{\mathrm{b}}$ may have significant advantages over the alternative KMC approach, where stochastic trajectories are followed from minimum b until they reach an A minimum. This methodology would provide the mean first-passage time from b to $\mathrm{A}, T_{\mathrm{b}}$, from which we could calculate the overall rate constants as

$$
k_{\mathrm{AB}}^{\mathrm{KMC}}=\frac{1}{P_{\mathrm{B}}^{\mathrm{eq}}} \sum_{\mathrm{b} \in \mathrm{B}} \frac{P_{\mathrm{b}}^{\mathrm{eq}}}{T_{\mathrm{b}}} \quad \text { and } \quad k_{\mathrm{BA}}^{\mathrm{KMC}}=\frac{1}{P_{\mathrm{A}}^{\mathrm{eq}}} \sum_{\mathrm{a} \in \mathrm{A}} \frac{P_{\mathrm{a}}^{\mathrm{eq}}}{T_{\mathrm{a}}} .
$$

However, if there is a large potential energy barrier between the A and B regions then the KMC trajectory will revisit minima in the B region many times before finally reaching an A minimum. $T_{\mathrm{b}}$ will then be dominated by a vast number of unsuccessful $b^{\prime} \leftarrow b$ transitions. $^{23}$ In contrast, the KMC trajectories employed to calculate $t_{\mathrm{b}}$ terminate when they hit either an A or a B minimum, and are generally very short when there is a large barrier. The vast majority of KMC trajectories return to the B region after a small number of steps in this situation. 
The rate constant formulations that employ committor probabilities in (6) and (8) will be more efficient that the expressions that use the mean first-passage time in (10) if we can calculate the $C_{\mathrm{b}}^{\mathrm{A}}$ or $C_{\mathrm{a}}^{\mathrm{B}}$ faster than $T_{\mathrm{b}}$ or $T_{\mathrm{a}}$. Our experience suggests that this is indeed the case, and that the corresponding speedup can be very large. Once the required committor probabilities are known it is also possible to compare the results from equations (6) and (8) to provide some measure of whether two-state kinetics are appropriate.

\section{3 'Leapfrog' Moves in Kinetic Monte Carlo}

At low temperatures we have found that the $\mathrm{KMC}$ runs used to calculate $T_{\mathrm{b}}$ and $t_{\mathrm{b}}$ may involve an inordinate number of recrossings for pairs of I minima separated by very low barriers. ${ }^{9}$ This problem was addressed in previous work by evaluating the escape probability and associated waiting time analytically for such pairs. ${ }^{9}$ A more general scheme was employed in the present work by calculating the probability and waiting times associated with jumps to second-neighbours for each local minimum ('leapfrog' moves). A further development involves an expansion of the A and B regions to include other local minima, which uses a disconnectivity graph analysis, ${ }^{10,30,31}$ as described in \$4.

Consider a particular minimum i, with adjacent minima (connected directly by a single transition state) labelled by an index $\mathrm{j}$, and second-neighbours labelled by index $\mathrm{k} \neq \mathrm{i}$. For all minima $\mathrm{j} \notin \mathrm{A} \cup \mathrm{B}$ we must allow for an arbitrary number of $\mathrm{i} \leftrightarrow \mathrm{j}$ recrossings before escape to a second neighbour, and the recrossings can occur in any order. Recrossings to minima $\mathrm{j} \in \mathrm{A} \cup \mathrm{B}$ are not allowed, but escape to such minima must be included. Transitions $\mathrm{i} \rightarrow \mathrm{j} \notin \mathrm{A} \cup \mathrm{B} \rightarrow \mathrm{k} \neq \mathrm{i}$ and $\mathrm{i} \rightarrow \mathrm{j} \in \mathrm{A} \cup \mathrm{B}$ can therefore be broken down into independent events, where the first event involves $\mathrm{i} \leftrightarrow \mathrm{j}$ recrossings and the second event involves the actual escape. The following derivation is not applicable if neighbour $\mathrm{j}$ has a direct connection to another minimum, $\mathrm{j}^{\prime}$, that is 
also adjacent to $\mathrm{i}$, since then we would need to account for arbitrary $\mathrm{j} \leftrightarrow \mathrm{j}^{\prime}$ recrossings as well. A more general scheme, which includes such effects, is described in \$5.

We now define new second neighbour branching probabilities, $P_{\mathrm{ki}}^{\prime}$, which include all possible recrossings between $\mathrm{i}$ and $\mathrm{j} \notin \mathrm{A} \cup \mathrm{B}$. Consider paths from $\mathrm{i}$ to $\mathrm{k}$ that include $n_{\mathrm{j}}$ recrossings $\mathrm{i} \leftrightarrow \mathrm{j}$ for each $\mathrm{j} \notin \mathrm{A} \cup \mathrm{B}$, with $n=\sum_{\mathrm{j}} n_{\mathrm{j}}$. The number of distinct paths of this kind is $n ! / \prod_{\mathrm{j}} n_{\mathrm{j}}$ !, and so the total probability of reaching $\mathrm{k}$ from $\mathrm{i}$ is

$$
\begin{aligned}
P_{\mathrm{ki}}^{\prime} & =\left(\sum_{\mathrm{j} \notin \mathrm{A} \cup \mathrm{B}} P_{\mathrm{kj}} P_{\mathrm{ji}}\right) \sum_{n=0}^{\infty} \sum_{n_{\mathrm{j}}}^{\prime}\left(n ! \prod_{\mathrm{j} \notin \mathrm{A} \cup \mathrm{B}}\left(P_{\mathrm{ij}} P_{\mathrm{ji}}\right)^{n_{\mathrm{j}}} / \prod_{\mathrm{j} \notin \mathrm{A} \cup \mathrm{B}} n_{\mathrm{j}} !\right) \\
& =\left(\sum_{\mathrm{j} \notin \mathrm{A} \cup \mathrm{B}} P_{\mathrm{kj}} P_{\mathrm{ji}}\right) \sum_{n=0}^{\infty}\left(\sum_{\mathrm{j} \notin \mathrm{A} \cup \mathrm{B}} P_{\mathrm{ij}} P_{\mathrm{ji}}\right)^{n} \\
& =\sum_{\mathrm{j} \notin \mathrm{A} \cup \mathrm{B}} P_{\mathrm{kj}} P_{\mathrm{ji}} /\left(1-\sum_{\mathrm{j} \notin \mathrm{A} \cup \mathrm{B}} P_{\mathrm{ij}} P_{\mathrm{ji}}\right),
\end{aligned}
$$

where we have used the multinomial theorem, ${ }^{32}$ and the prime for the sum over $n_{\mathrm{j}}$ indicates a restriction to $n_{\mathrm{j}} \geq 0$ and $\sum_{\mathrm{j}} n_{\mathrm{j}}=n$, with $\mathrm{j} \notin \mathrm{A} \cup \mathrm{B}$. Similarly, the total probability of reaching a minimum $j \in \mathrm{A} \cup \mathrm{B}$ from $\mathrm{i}$ is

$$
P_{\mathrm{ji}}^{\prime}=P_{\mathrm{ji}} /\left(1-\sum_{\mathrm{j} \notin \mathrm{A} \cup \mathrm{B}} P_{\mathrm{ij}} P_{\mathrm{ji}}\right)
$$

It is easily verified that the new branching probabilities out of i sum to unity, as they should.

The mean waiting time for a transition from i to any second neighbour, $\mathrm{k}$, or adjacent minimum, $\mathrm{j} \in \mathrm{A} \cup \mathrm{B}$, is written as $\tau_{\mathrm{i}}^{\prime}$. To calculate $\tau_{\mathrm{i}}^{\prime}$ we note that every step associated with a branching probability $P_{\alpha \beta}$ adds $\tau_{\beta}$ to the duration of a path, on average. If we replace each branching probability $P_{\alpha \beta}$ by $\widetilde{P}_{\alpha \beta}=P_{\alpha \beta} \exp \left(\zeta \tau_{\beta}\right)$ then the time associated with any path, multiplied by its probability, can be obtained from

$$
\left[\frac{d}{d \zeta} \widetilde{P}_{\alpha_{1} \alpha_{2}} \widetilde{P}_{\alpha_{2} \alpha_{3}} \widetilde{P}_{\alpha_{3} \alpha_{4}} \ldots \widetilde{P}_{\alpha_{n-1} \alpha_{n}}\right]_{\zeta=0}=P_{\alpha_{1} \alpha_{2}} P_{\alpha_{2} \alpha_{3}} P_{\alpha_{3} \alpha_{4}} \ldots P_{\alpha_{n-1} \alpha_{n}}\left(\tau_{\alpha_{2}}+\tau_{\alpha_{3}}+\ldots+\tau_{\alpha_{n}}\right)
$$


Hence

$$
\begin{aligned}
\tau_{i}^{\prime} & =\left[\frac{d}{d \zeta}\left(\sum_{\mathrm{k}} \sum_{\mathrm{j} \notin \mathrm{A} \cup \mathrm{B}} \widetilde{P}_{\mathrm{kj}} \widetilde{\mathrm{j}}_{\mathrm{ji}}+\sum_{\mathrm{j} \in \mathrm{A} \cup \mathrm{B}} \widetilde{P}_{\mathrm{ji}}\right) /\left(1-\sum_{\mathrm{j} \notin \mathrm{A} \cup \mathrm{B}} \widetilde{P}_{\mathrm{ij}} \widetilde{\mathrm{j}}_{\mathrm{ji}}\right)\right]_{\zeta=0} \\
& =\left(\tau_{\mathrm{i}}+\sum_{\mathrm{j} \notin \mathrm{A} \cup \mathrm{B}} \tau_{\mathrm{j}} P_{\mathrm{ji}}\right) /\left(1-\sum_{\mathrm{j} \notin \mathrm{A} \cup \mathrm{B}} P_{\mathrm{ij}} P_{\mathrm{ji}}\right) .
\end{aligned}
$$

In the present work $\tau_{\mathrm{i}}^{\prime}$ and the corresponding leapfrog transition probabilities, $P_{\alpha \mathrm{i}}^{\prime}$, were calculated before the KMC phase of the calculation for each minimum with no direct connections between its first neighbours. Leapfrog moves were used for eligible minima in the I set if any branching probability $P_{\alpha \mathrm{i}}$ exceeded a threshold value. In practice, thresholds ranging from 0.1 to 0.8 were found to work equally well, and gave essentially identical results. The corresponding speedup can be very large, as discussed in $\sqrt[6]{6}$

\section{Reclassification of the A, B and I Local Minima}

When reanalysing stationary point databases obtained in previous work, ${ }^{9,11}$ situations were still encountered where the $\mathrm{KMC}$ runs became stuck in extended regions consisting of local minima separated by small barriers. This observation suggests that minima in the $\mathrm{B}$ region (for $\mathrm{A} \leftarrow \mathrm{B}$ rates) are connected to relatively low-lying I minima by barriers that are smaller than those for return to a B minimum. Expanding the B region to include such minima has a negligible effect on the two-state rate constants. In the present work we have defined expansions of the B (and A) regions using the same superbasin analysis that is employed in constructing disconnectivity graphs. ${ }^{10,30,31}$ The local minima are partitioned into disjoint sets (superbasins), so that at least one pathway exists between any two minima of each set that does not exceed a threshold energy, $E_{\text {th }}$. In contrast, any path between minima in different sets must include a transition state that lies above $E_{\mathrm{th}}$. For a given $E_{\mathrm{th}}$, all local minima in the same superbasin as a B minimum were classified as $\mathrm{B}$, and similarly for the A region. When the threshold, 
$E_{\text {th }}$, is low enough, this procedure results in no reclassifications; there is also a maximum threshold above which A and B minima would share the same superbasin. For the databases considered below, the threshold could be varied over quite a wide range without changing the rate constants by more than about a factor of two. However, the corresponding CPU time can vary by orders of magnitude, as described below.

\section{The Graph Transformation Method}

The main idea of the graph transformation approach is to progressively remove local minima from the I set whilst leaving the average properties of interest unchanged for the database that remains. The theory is an extension of the results used to perform jumps to second neighbours in previous KMC simulations, ${ }^{9}$ and the leapfrog moves considered in 93 , which themselves share some common ground with Novotny's 'Monte Carlo with absorbing Markov chains' approach. ${ }^{33}$ The present method extends the approach of Bortz, Kalos and Lebowitz ${ }^{19}$ to exclude not only the transitions from the current state into itself but also transitions involving an adjacent minimum. For example, suppose we wish to remove minimum $\mathrm{i} \in \mathrm{I}$. Consider KMC trajectories that arrive at minimum $\beta$, which is adjacent to $\mathrm{i}$. We wish to step directly from $\beta$ to any minimum in the set $\Gamma$ that is adjacent to $\beta$ or $\mathrm{i}$, excluding these two minima themselves. To ensure that the mean first-passage times between the A and B sets are unchanged we must define new branching probabilities, $P_{\gamma \beta}^{\prime}$ from $\beta$ to all $\gamma \in \Gamma$, along with a new waiting time for escape from $\beta, \tau_{\beta}^{\prime}$. Here, $\tau_{\beta}^{\prime}$ corresponds to the mean waiting time for escape from $\beta$ to any $\gamma \in \Gamma$, while the modified branching probabilities subsume all the possible recrossings involving minimum $\beta$ that could occur before a transition to a minimum in $\Gamma$. Hence the new branching probabilities are:

$$
P_{\gamma \beta}^{\prime}=\left(P_{\gamma \mathrm{i}} P_{\mathrm{i} \beta}+P_{\gamma \beta}\right) \sum_{m=0}^{\infty}\left(P_{\beta \mathrm{i}} P_{\mathrm{i} \beta}\right)^{m}=\left(P_{\gamma \mathrm{i}} P_{\mathrm{i} \beta}+P_{\gamma \beta}\right) /\left(1-P_{\beta \mathrm{i}} P_{\mathrm{i} \beta}\right)
$$


This formula also applies if either $P_{\gamma \beta}$ or $P_{\gamma i}$ vanishes. When calculating mean firstpassage times for transitions from the $\mathrm{B}$ to $\mathrm{A}$ regions we do not consider branching probabilities out of A minima, and similarly for branching probabilities out of B minima when considering $\mathrm{A}$ to $\mathrm{B}$ transitions. However, $P_{\gamma \beta}^{\prime}$ and $\tau_{\beta}^{\prime}$ for $\beta \in \mathrm{A}$ are never used in calculating the $\mathrm{A} \leftarrow \mathrm{B}$ rates, and similarly for $\beta \in \mathrm{B}$ and $\mathrm{B} \leftarrow \mathrm{A}$ rates. Hence we can use equation (14) for all minima $\beta \in \mathrm{A} \cup \mathrm{B} \cup \mathrm{I}$, and obtain results for both $\mathrm{A} \leftarrow \mathrm{B}$ and $\mathrm{B} \leftarrow \mathrm{A}$ rate constants at the end of the procedure. Detailed balance can then be used as a consistency check.

It is easy to show that the new branching probabilities are normalised:

$$
\sum_{\gamma \in \Gamma} \frac{P_{\gamma \mathrm{i}} P_{\mathrm{i} \beta}+P_{\gamma \beta}}{1-P_{\beta \mathrm{i}} P_{\mathrm{i} \beta}}=\frac{\left(1-P_{\beta \mathrm{i}}\right) P_{\mathrm{i} \beta}+\left(1-P_{\mathrm{i} \beta}\right)}{1-P_{\beta \mathrm{i}} P_{\mathrm{i} \beta}}=1 .
$$

To calculate $\tau_{\beta}^{\prime}$ we use the method of Sec. B.

$$
\tau_{\beta}^{\prime}=\left[\frac{d}{d \zeta} \sum_{\gamma \in \Gamma} \frac{P_{\gamma \mathrm{i}} P_{\mathrm{i} \beta} e^{\zeta\left(\tau_{\mathrm{i}}+\tau_{\beta}\right)}+P_{\gamma \beta} e^{\zeta \tau_{\beta}}}{1-P_{\beta \mathrm{i}} P_{\mathrm{i} \beta} e^{\zeta\left(\tau_{\mathrm{i}}+\tau_{\beta}\right)}}\right]_{\zeta=0}=\frac{\tau_{\beta}+P_{\mathrm{i} \beta} \tau_{\mathrm{i}}}{1-P_{\beta \mathrm{i}} P_{\mathrm{i} \beta}} .
$$

The modified branching probabilities and waiting times could be used in a KMC simulation based upon the stationary point database with minimum i excluded. Since the modified branching probabilities, $P_{\gamma \beta}^{\prime}$, subsume the sums over all paths from $\beta$ to $\gamma$ that involve $i$ we would expect the probability that a trajectory starting at $\mathrm{b} \in \mathrm{B}$ and ending at a $\in \mathrm{A}$ is conserved. Here we consider for specificity the $k_{\mathrm{AB}}$ rate constant, corresponding to paths that start from B minima and terminate at an A minimum. In contrast to the formulation of $k_{\mathrm{AB}}^{\mathrm{SS}}$ and $k_{\mathrm{AB}}^{\mathrm{NSS}}$, above, revisits to $\mathrm{B}$ minima are now allowed, as for the conventional KMC calculations that yield $k_{\mathrm{AB}}^{\mathrm{KMC}}$ in equation (10). Since each trajectory exiting from $\gamma \in \Gamma$ acquires a time increment equal to the average value, $\tau_{\beta}^{\prime}$, the contributions to the mean first-passage time from individual A minima are not conserved (unless there is a single A minimum). Nevertheless, the overall mean first-passage time to $\mathrm{A}$ is conserved, i.e. $\mathcal{T}_{\mathrm{b}}=\mathcal{T}_{\mathrm{b}}^{\prime}$, where the prime denotes a transformed quantity. To prove these results consider the effect of removing minimum i on trajectories reaching minimum $\beta$, which is initially connected to i, from minimum 
$\mathrm{b} \in \mathrm{B}$. The total probability of a pathway terminating at $\mathrm{a}$ if it starts from $\mathrm{b}$ is the sum of the product of branching probabilities, $\mathcal{W}_{\xi}=P_{\mathrm{a} \xi_{\mathrm{n}}} P_{\xi_{n} \xi_{n-1}} \ldots P_{\xi_{2} \xi_{1}} P_{\xi_{1} \mathrm{~b}}$, over all the corresponding paths $\xi \in \mathrm{a} \leftarrow \mathrm{b}$ :

$$
\begin{aligned}
\sum_{\xi \in \mathrm{a} \leftarrow \mathrm{b}} \mathcal{W}_{\xi} & =\sum_{\xi_{3} \in \Xi^{\prime}} \mathcal{W}_{\xi_{3}}+\sum_{\xi_{1} \in \beta \leftarrow b} \mathcal{W}_{\xi_{1}} \sum_{\gamma \in \Gamma}\left(P_{\gamma \beta}+P_{\gamma \mathrm{i}} P_{\mathrm{i} \beta}\right) \sum_{m=0}^{\infty}\left(P_{\beta \mathrm{i}} P_{i \beta}\right)^{m} \sum_{\xi_{2} \in a \leftarrow \gamma} \mathcal{W}_{\xi_{2}} \\
& =\sum_{\xi_{3} \in \Xi^{\prime}} \mathcal{W}_{\xi_{3}}+\sum_{\xi_{1} \in \beta \leftarrow \mathrm{b}} \mathcal{W}_{\xi_{1}} \sum_{\gamma \in \Gamma} P_{\gamma \beta}^{\prime} \sum_{\xi_{2} \in \mathrm{a} \leftarrow \gamma} \mathcal{W}_{\xi_{2}},
\end{aligned}
$$

and similarly for any other minimum adjacent to i and any other pathway that revisits $\beta$ other than by immediate recrossings of the type $\beta \rightarrow \mathrm{i} \rightarrow \beta$. $\Xi$ is the ensemble of all paths for which probabilistic weights cannot be written in the form defined by the last term of the above equation. Here $\Xi^{\prime}$ is the ensemble of all paths which probabilistic weights cannot be written in the form defined by the last term of the above equation. Hence the transformation preserves the individual probabilities $\sum_{\xi \in \mathrm{a} \leftarrow \mathrm{b}} \mathcal{W}_{\xi}$.

Now consider the effect of removing minimum i on the contribution to the mean first-passage time from $b \in \mathrm{B}$ to A using the approach of Sec. 3

$$
\begin{aligned}
& {\left[\frac{d}{d \zeta} \sum_{\xi_{1} \in \beta \leftarrow \mathrm{b}} \widetilde{\mathcal{W}}_{\xi_{1}} \sum_{\gamma \in \Gamma} \widetilde{P}_{\gamma \beta}^{\prime} \sum_{\xi_{2} \in a \leftarrow \gamma} \widetilde{\mathcal{W}}_{\xi_{2}}\right]_{\zeta=0} } \\
= & \sum_{\xi_{1} \in \beta \leftarrow \mathrm{b}}\left[\frac{d \widetilde{\mathcal{W}}_{\xi_{1}}}{d \zeta}\right]_{\zeta=0} \sum_{\gamma \in \Gamma} P_{\gamma \beta}^{\prime} \sum_{\xi_{2} \in a \leftarrow \gamma} \mathcal{W}_{\xi_{2}} \\
& +\sum_{\xi_{1} \in \beta \leftarrow \mathrm{b}} \mathcal{W}_{\xi_{1}} \sum_{\gamma \in \Gamma}\left[\frac{d \widetilde{P}_{\gamma \beta}^{\prime}}{d \zeta}\right]_{\zeta=0} \sum_{\xi_{2} \in a \leftarrow \gamma} \mathcal{W}_{\xi_{2}} \\
& +\sum_{\xi_{1} \in \beta \leftarrow \mathrm{b}} \mathcal{W}_{\xi_{1}} \sum_{\gamma \in \Gamma} P_{\gamma \beta}^{\prime} \sum_{\xi_{2} \in \mathrm{a} \leftarrow \gamma}\left[\frac{d \widetilde{\mathcal{W}}_{\xi_{2}}}{d \zeta}\right]_{\zeta=0},
\end{aligned}
$$

where the tildes indicate that every branching probability $P_{\alpha \beta}$ is replaced by $P_{\alpha \beta} e^{\zeta \tau_{\beta}}$, as in $\$ 3$. The first and last terms are unchanged from the original database in this construction, but the middle term,

$$
\begin{aligned}
& \sum_{\xi_{1} \in \beta \leftarrow b} \mathcal{W}_{\xi_{1}} \sum_{\gamma \in \Gamma}\left[\frac{d \widetilde{P}_{\gamma \beta}^{\prime}}{d \zeta}\right]_{\zeta=0} \sum_{\xi_{2} \in \mathrm{a} \leftarrow \gamma} \mathcal{W}_{\xi_{2}} \\
= & \sum_{\xi_{1} \in \beta \leftarrow b} \mathcal{W}_{\xi_{1}} \sum_{\gamma \in \Gamma} \frac{P_{\gamma \mathrm{i}} P_{\mathrm{i} \beta}\left(\tau_{\beta}+\tau_{\mathrm{i}}\right)+P_{\gamma \beta}\left(\tau_{\beta}+P_{\beta \mathrm{i}} P_{i \beta} \tau_{\mathrm{i}}\right)}{\left(1-P_{\beta \mathrm{i}} P_{\mathrm{i} \beta}\right)^{2}} \sum_{\xi_{2} \in \mathrm{a} \leftarrow \gamma} \mathcal{W}_{\xi_{2}},
\end{aligned}
$$


is different (unless there is only one A minimum). However, if we sum over A minima

then $\sum_{\mathrm{a} \in \mathrm{A}} \sum_{\xi_{2} \in \mathrm{a} \leftarrow \gamma} \widetilde{\mathcal{W}}_{\xi_{2}}=1$ for all $\gamma$, and we can now simplify the sum over $\gamma$ as

$$
\sum_{\gamma \in \Gamma} \frac{P_{\gamma \mathrm{i}} P_{\mathrm{i} \beta}\left(\tau_{\beta}+\tau_{\mathrm{i}}\right)+P_{\gamma \beta}\left(\tau_{\beta}+P_{\beta \mathrm{i}} P_{\mathrm{i} \beta} \tau_{\mathrm{i}}\right)}{\left(1-P_{\beta \mathrm{i}} P_{\mathrm{i} \beta}\right)^{2}}=\tau_{\beta}^{\prime}=\sum_{\gamma \in \Gamma} P_{\gamma \beta}^{\prime} \tau_{\beta}^{\prime} .
$$

The same argument can be applied whenever a trajectory reaches a minimum adjacent to $\mathrm{i}$, so that $\mathcal{T}_{\mathrm{b}}^{\prime}=\mathcal{T}_{\mathrm{b}}$, as required.

At low temperature some of the $P_{\alpha \beta}$ approach unity, and numerical problems arise in calculating terms like $1-P_{\alpha \beta} P_{\beta \alpha}$. However, precision can be regained by writing

$$
P_{\alpha \beta}=1-\sum_{\gamma \neq \alpha} P_{\gamma \beta} \equiv 1-\epsilon_{\alpha \beta} \quad \text { and } \quad P_{\beta \alpha}=1-\sum_{\gamma \neq \beta} P_{\gamma \alpha} \equiv 1-\epsilon_{\beta \alpha},
$$

and then using $1-P_{\alpha \beta} P_{\beta \alpha}=\epsilon_{\alpha \beta}-\epsilon_{\alpha \beta} \epsilon_{\beta \alpha}+\epsilon_{\beta \alpha}$.

Further applications of the graph transformation method will be described elsewhere in the context of Markov chains considered as digraphs. ${ }^{34}$ In particular, we note that more general formulations are possible so that waiting times involving revisits to arbitrary sets of minima can be included. The main advantage of this theory is that there is an upper bound to the maximum operation count involved in removing all the I minima. In contrast to the successive overrelaxation technique used to calculate committor probabilities, described in 92 , the graph transformation does not depend upon satisfying any convergence criteria. Once all the I minima have been removed the only transformed branching probabilities remaining, $P_{\mathrm{ab}}^{\prime}$, are the relative probabilities of paths from $\mathrm{A}$ and $\mathrm{B}$ minima terminating at any of the other $\mathrm{A}$ and $\mathrm{B}$ minima. The final waiting times, $\tau_{\mathrm{b}}^{\prime}$, correspond to the average time before a transition to any other A or B minimum starting from b, and similarly for the $\tau_{\mathrm{a}}^{\prime}$. Here we use a single prime to denote the final values of transformed quantities, although in general they may change many times as the I minima are successively removed. The associated rate constants can then be calculated as

$$
k_{\mathrm{AB}}^{\mathrm{GT}}=\frac{1}{P_{\mathrm{B}}^{\mathrm{eq}}} \sum_{\mathrm{b} \in \mathrm{B}} \frac{P_{\mathrm{b}}^{\mathrm{eq}}}{\tau_{\mathrm{b}}^{\prime}} \sum_{\mathrm{a} \in \mathrm{A}} P_{\mathrm{ab}}^{\prime} \quad \text { and } \quad k_{\mathrm{BA}}^{\mathrm{GT}}=\frac{1}{P_{\mathrm{A}}^{\mathrm{eq}}} \sum_{\mathrm{a} \in \mathrm{A}} \frac{P_{\mathrm{a}}^{\mathrm{eq}}}{\tau_{\mathrm{a}}^{\prime}} \sum_{\mathrm{b} \in \mathrm{B}} P_{\mathrm{ba}}^{\prime} .
$$


The transformed probabilities and waiting times in equation (22) are different from the committor probabilities and waiting times that appear in the definitions of $k_{\mathrm{AB}}^{\mathrm{SS}}$ and $k_{\mathrm{AB}}^{\mathrm{NSS}}$, and from the waiting times that appear in equation (10) for $k_{\mathrm{AB}}^{\mathrm{KMC}}$. It is also noteworthy that we have avoided the steady-state approximation for the I minima. Since the computational cost of the graph transformation does not change as the temperature decreases, in contrast to the successive overrelaxation calculations and conventional KMC runs, this approach becomes increasingly advantageous at low temperature. In fact it is also possible to define transformations that enable us to evaluate $k_{\mathrm{AB}}^{\mathrm{NSS}}$ and $k_{\mathrm{AB}}^{\mathrm{KMC}}$ precisely in a fixed number of operations; these results will be presented elsewhere. $^{34}$

\section{Results}

The formulations in equations (6), (8), and (22) were tested for stationary point databases obtained in previous DPS runs for permutational isomerization and morphological transitions of four different atomic clusters bound by the Lennard-Jones (LJ) potential. ${ }^{35}$ In each case Arrhenius fits of $k_{\mathrm{AB}}$ and $k_{\mathrm{BA}}$ were presented in previous work, ${ }^{9}$ and only for the cluster containing 55 atoms, $\mathrm{LJ}_{55}$, are the results significantly different here. For the three other examples $\left(\mathrm{LJ}_{13}, \mathrm{LJ}_{38}\right.$ and $\left.\mathrm{LJ}_{75}\right)$ the effective barrier heights changed by less than $0.05 \epsilon$, and the Arrhenius prefactors changed by a factor of three or less. Here $\epsilon$ is the pair well depth for the LJ potential. The new results for $\mathrm{LJ}_{55}$ are therefore considered in more detail below.

The global potential energy minimum for $\mathrm{LJ}_{55}$ is a Mackay icosahedron, ${ }^{36}$ which exhibits special stability and 'magic number' properties. ${ }^{37,38}$ There are four distinct sites for a tagged atom in the global minimum: one in the centre, one in the middle shell, and two in the outer shell. ${ }^{9}$ DPS calculations in previous work considered the rate for migration of the tagged atom between the centre and either one of the surface sites. ${ }^{10}$ Even at the solid-like/liquid-like melting transition temperature for this 
cluster the potential energy barrier between these $\mathrm{A}$ and $\mathrm{B}$ states is around $50 k_{B} T$. Temperatures down to $k_{B} T / \epsilon=0.04$ were considered in the present work, where the barrier is $350 k_{B} T$. Standard KMC calculations for this system previously proved to be unfeasible below about $k_{B} T / \epsilon=0.3 .^{9}$

A disconnectivity graph that distinguishes permutation-inversion isomers of the tagged atom is shown in Figure 1. Separate branches appear for the two distinct surface sites, but these minima were grouped together in state B for the rate constant calculations. The branch for group A, at the right of the graph, has the tagged atom at the centre of the icosahedron. The calculated two-state rate constants do not vary significantly over a wide range of $E_{\mathrm{th}}$, and $\mathrm{KMC}$ averages for $t_{\mathrm{b}}$ employing 1000 trajectories are generally sufficient. For higher thresholds the A and B sets start to overlap, indicating that the highest energy transition state on the lowest energy path between the $\mathrm{A}$ and $\mathrm{B}$ regions lies approximately $14 \epsilon$ above the global minimum. Using a fractional convergence tolerance of $10^{-3}$ for the total rate in the committor probability calculations and KMC averages over 1000 trajectories per B minimum, the entire analysis of the database requires only a few seconds of CPU time on an UltraSparcIII $900 \mathrm{MHz}$ processor.

The temperature dependence of both $k_{\mathrm{AB}}^{\mathrm{NSS}}$ and $k_{\mathrm{BA}}^{\mathrm{NSS}}$, for the surface-to-centre and centre-to-surface rates, can be fitted quite accurately (coefficient of determination, $\left.R^{2}=0.99999\right)$ by the Arrhenius form $k=a \exp \left(-\Delta / k_{B} T\right)$ for $0.04 \leq k_{B} T / \epsilon \leq 0.3$ :

$$
\begin{aligned}
& k_{\mathrm{AB}}: \quad a=1.38 \times 10^{8} \nu_{\mathrm{LJ}}, \quad \Delta=14.05 \epsilon, \\
& k_{\mathrm{BA}}: \quad a=6.52 \times 10^{9} \nu_{\mathrm{LJ}}, \quad \Delta=14.04 \epsilon,
\end{aligned}
$$

where the unit of frequency is $\nu_{\mathrm{LJ}}=\sqrt{\epsilon / M \sigma^{2}}$, with $M$ the atomic mass and $2^{1 / 6} \sigma$ the equilibrium pair separation. Symmetry requires that $42 k_{\mathrm{AB}}=k_{\mathrm{BA}}$ when only permutation-inversion isomers of the global minimum including a tagged atom are included in the $\mathrm{A}$ and $\mathrm{B}$ sets. ${ }^{9}$ The above results deviate a little from this ideal ratio because there are 77 minima in the $\mathrm{A}$ set and 1763 minima in the $\mathrm{B}$ set using 
reclassification at a threshold energy of $E_{\text {th }}=-270 \epsilon$. At $k_{B} T / \epsilon=0.1$ the centre-tosurface rate constant has order $10^{-40} \mathrm{~s}^{-1}$ for parameters appropriate to argon.

The above rates are significantly faster than those obtained from a summation over DPS paths in previous work,${ }^{9}$ indicating that the latter sums were not converged. In particular, the value of the effective barrier, $\Delta$, in the Arrhenius fit is about $4 \epsilon$ lower, and now coincides closely with the barrier corresponding to the highest transition state on the lowest energy path between the A and B regions. The present results therefore supersede the values obtained in reference [11].

A more detailed analysis of the results for $\mathrm{LJ}_{55}$ (Tables 1,4) reveals a number of trends that are likely to be useful in future work, especially regarding the choice of $E_{\text {th }}$ and two-state kinetics. At $k_{B} T / \epsilon=0.4$ we find that $k_{\mathrm{AB}}^{\mathrm{SS}}, k_{\mathrm{AB}}^{\mathrm{NSS}}$ and $k_{\mathrm{AB}}^{\mathrm{GT}}$ agree well for $-267 \epsilon \geq E_{\mathrm{th}} \geq-270 \epsilon$ (Table 1 ). These values also agree with $k_{\mathrm{AB}}^{\mathrm{KMC}}$ calculated without any regrouping, although the latter simulation requires about a thousand times more CPU time. It is also interesting to note that the calculated values in Table 1 exhibit jumps when $E_{\text {th }}$ changes from $-270 \epsilon$ to $-271 \epsilon$, from $-273 \epsilon$ to $-274 \epsilon$, and from $-275 \epsilon$ to $-276 \epsilon$. Changes in behaviour occur at the same points for lower temperatures, as described below, and the cause is easily identified with the aid of Figure 1] For $E_{\mathrm{th}}=-270 \epsilon$ the Mackay icosahedra corresponding to both minima in the original B set, and the $C_{5 v}$ minimum with the tagged atom in the intermediate shell, all lie in the same superbasin. According to the reclassification scheme, all the local minima belonging to this superbasin are then classified as type B. However, at $E_{\mathrm{th}}=-271 \epsilon$ the branch corresponding to the tagged atom in the intermediate shell splits off from the branch containing the original $\mathrm{B}$ minima, so there is a qualitative change in the classification of local minima between these energies. Nevertheless, the overall $\mathrm{A} \leftrightarrow \mathrm{B}$ kinetics are not disturbed dramatically, because equilibrium between the remaining B minima and the region that is now classed as 'intervening' is still established rapidly compared to the time scale for transitions between A and B. As Figure 1 clearly shows, 
the corresponding potential energy barrier for $\mathrm{A} \leftrightarrow \mathrm{B}$ interconversion is about $5 \epsilon$ larger than for equilibration with this I region. It is also noteworthy that the relation $42 k_{\mathrm{AB}}=$ $k_{\mathrm{BA}}$ is obeyed to better than $0.2 \%$ for $E_{\mathrm{th}}=-271 \epsilon$, because the A and B sets are not expanded as much as for $E_{\mathrm{th}}=-270 \epsilon$, where the Arrhenius fit was performed (above).

On changing $E_{\text {th }}$ from $-273 \epsilon$ to $-274 \epsilon$ the two icosahedra with the tagged atom in the surface separate into different superbasins. Some local minima corresponding to icosahedra with a surface vacancy and adatom pair also separate into their own branches, and are therefore assigned to the I set. This regrouping again affects $k_{\mathrm{AB}}^{\mathrm{SS}}$, $k_{\mathrm{AB}}^{\mathrm{NSS}}$, and $k_{\mathrm{AB}}^{\mathrm{GT}}$, but the difference is only a factor of about two. Nevertheless, the change in $k_{\mathrm{AB}}^{\mathrm{NSS}}$ below $E_{\mathrm{th}}=-270 \epsilon$ suggests that a two-state description is less appropriate for the classification of A, B and I sets corresponding to lower energy thresholds.

When the temperature is lowered to $k_{B} T / \epsilon=0.3$ we again see changes in the calculated rate constants at the same $E_{\mathrm{th}}$ boundaries as for $k_{B} T / \epsilon=0.4$. However, there is one important change from the results at $k_{B} T / \epsilon=0.4$. A much tighter convergence condition is required in the calculation of committor probabilities for $E_{\mathrm{th}} \leq$ $-271 \epsilon$ at the lower temperature, and the required CPU time jumps by a factor of about 100. This effect can again be explained from the corresponding classification of the A, B and I sets with reference to the disconnectivity graph. The committor probability calculation requires many more iterations to converge for $E_{\mathrm{th}} \leq-271 \epsilon$ because there are I minima with very low values of $C_{\mathrm{i}}^{\mathrm{A}}$. The relative $\mathrm{CPU}$ time required to calculate $k_{\mathrm{AB}}^{\mathrm{GT}}$ is now significantly smaller, since it is essentially unchanged from $k_{B} T / \epsilon=0.4$.

For $k_{B} T / \epsilon=0.2$ the benefit of leapfrog KMC moves becomes visible (Table [3), producing a speedup factor of between ten and a few hundred. An even tighter condition is required to converge the committor probability calculation for $E_{\text {th }} \leq-271 \epsilon$, with a corresponding dramatic increase in the time required. At $k_{B} T / \epsilon=0.1$ (Table 4) KMC runs without leapfrog moves were not feasible, and only $k_{\mathrm{AB}}^{\mathrm{GT}}$ could be calculated for $E_{\mathrm{th}} \leq-271 \epsilon$. Note that the timings for $k_{\mathrm{AB}}^{\mathrm{GT}}$ are practically independent of 
temperature, which makes the graph transformation approach highly advantageous at low temperatures.

\section{Conclusions}

In this contribution we have shown how the original discrete path sampling (DPS) rate expressions, which involve the steady-state approximation for 'intervening' local minima, can be written in terms of committor probabilities. Analogous expressions can be derived without the steady-state approximation by simply changing the waiting time associated with escape from each $\mathrm{B}\left(\right.$ for $k_{\mathrm{AB}}$ ) or $\mathrm{A}$ (for $\left.k_{\mathrm{BA}}\right)$ minimum. In the steadystate expressions the appropriate waiting times correspond to transitions to directly connected minima, involving a single transition state. In contrast, when the steadystate approximation is removed, the required waiting times correspond to transitions to any member of the $\mathrm{A}$ or $\mathrm{B}$ sets, and can be calculated from relatively short KMC runs. If the waiting times for escape from each intervening local minima to an adjacent minimum are small then the steady-state limit is recovered, as expected.

Committor probabilities can be calculated using successive overrelaxation techniques, and this approach is significantly faster than the direct sum over paths of increasing length used in previous work to calculate $k_{\mathrm{AB}}^{\mathrm{SS}}$ and $k_{\mathrm{BA}}^{\mathrm{SS}} \cdot{ }^{9,11}$ The KMC runs required to calculate waiting times for transitions to either the $\mathrm{A}$ or $\mathrm{B}$ region, and hence $k_{\mathrm{AB}}^{\mathrm{NSS}}$ or $k_{\mathrm{BA}}^{\mathrm{NSS}}$, are generally very short. In contrast, direct KMC simulations based on mean first-passage times involve trajectories that can only terminate in the product region. When the A and B states are separated by a high potential energy barrier the latter trajectories will generally revisit the reactant region many times. The alternative formulations based upon committor probabilities may then be advantageous, but require leapfrog KMC moves and reclassification of the A, B and I sets to be feasible at low temperatures.

The most powerful method that we have found for extracting phenomenological rate 
constants is the graph transformation approach. Intervening local minima are succes-

sively removed, and the branching probabilities and waiting times for the remaining local minima are renormalised to preserve the mean first-passage time between the A and $\mathrm{B}$ regions. At the end of the transformation only $\mathrm{A}$ and $\mathrm{B}$ minima remain, and the waiting time in each one is the average value for a transition to any of the other $\mathrm{A}$ and $\mathrm{B}$ minima. The advantage of this approach is that the time taken to perform the transformation does not depend upon temperature, so it can be used when the processes of interest are arbitrarily slow.

The main conclusion that we draw from the detailed analysis of $\mathrm{LJ}_{55}$ in 6 is that the threshold energy, $E_{\mathrm{th}}$, for reclassification of $\mathrm{A}$ and $\mathrm{B}$ minima should be chosen above the potential energy where any likely kinetic traps branch off. This choice of threshold should not affect the two-state dynamics of interest significantly, so long as the potential energy difference between the top of any possible traps and the highest transition state on the lowest $\mathrm{A} \leftrightarrow \mathrm{B}$ path is large compared to $k_{B} T$. In this case there will be a clear separation of time scales for relaxation between the A and B regions and within the regions themselves, so that a two-state description is still applicable. ${ }^{39,40}$ The disconnectivity graph approach ${ }^{30,31}$ provides a helpful way to recognise such situations. ${ }^{10}$ Both the graph transformation theory and leapfrog KMC moves combined with an appropriate choice of $E_{\text {th }}$ can produce exponential speedups in the kinetic analysis, providing access to temperatures where the potential energy barrier is more than a hundred times larger than $k_{B} T$.

\section{References}

[1] T. S. van Erp, D. Moroni and P. G. Bolhuis, J. Chem. Phys. 118, 7762 (2003).

[2] C. Dellago, P. Bolhuis and P. L. Geissler, Advances Chem. Phys. 123, 1 (2002). 
[3] P. G. Bolhuis, D. Chandler, C. Dellago and P. L. Geissler, Annu. Rev. Phys. Chem. 53, 291 (2002).

[4] N. Singhal, C. D. Snow and V. S. Pande, J. Chem. Phys. 121, 415 (2004).

[5] A. K. Faradjian and R. Elber, J. Chem. Phys. 120, 10880 (2004).

[6] S. Sriraman, I. G. Kevrekidis and G. Hummer, J. Phys. Chem. B 109, 6479 (2005).

[7] K. N. Kudin and R. Car, J. Chem. Phys. 122, 114108 (2005).

[8] A. Berezhkovskii and A. Szabo, J. Chem. Phys. 121, 9186 (2004).

[9] D. J. Wales, Mol. Phys. 100, 3285 (2002).

[10] D. J. Wales, Energy Landscapes: Applications to Clusters, Biomolecules and Glasses, Cambridge University Press, Cambridge (2003).

[11] D. J. Wales, Mol. Phys. 102, 883 (2004).

[12] F. H. Stillinger and T. A. Weber, Science 225, 983 (1984).

[13] R. E. Kunz and R. S. Berry, J. Chem. Phys. 103, 1904 (1995).

[14] J. N. Murrell and K. J. Laidler, Trans. Faraday Soc. 64, 371 (1968).

[15] N. G. van Kampen, Stochastic Processes in Physics and Chemistry, Elsevier, Amsterdam (1981).

[16] R. E. Kunz, Dynamics of First-Order Phase Transitions, Deutsch, Thun (1995).

[17] D. J. Wales and J. P. K. Doye, J. Chem. Phys. 119, 12409 (2003).

[18] W. H. Press, B. P. Flannery, S. A. Teukolsky and W. T. Vetterling, Numerical Recipes in Fortran: The Art of Scientific Computing, Cambridge University Press, Cambridge (1992).

[19] A. B. Bortz, M. H. Kalos and J. L. Lebowitz, J. Comput. Phys. 17, 10 (1975). 
[20] A. F. Voter, Phys. Rev. B 34, 6819 (1986).

[21] K. A. Fichthorn and W. H. Weinberg, J. Chem. Phys 95, 1090 (1991).

[22] D. A. Evans and D. J. Wales, J. Chem. Phys. 119, 9947 (2003).

[23] W. Cai, M. H. Kalos, M. de Koning and V. V. Bulatov, Phys. Rev. E 66, 046703 (2002).

[24] M. de Koning, W. Cai, B. Sadigh, T. Oppelstrup, M. H. Kalos and V. V. Bulatov, J. Chem. Phys. 122, 074103 (2005).

[25] R. Du, V. S. Pande, A. Y. Grosberg, T. Tanaka and E. I. Shakhnovich, J. Chem. Phys. 108, 334 (1998).

[26] C. D. Snow, E. J. Sorin, Y. M. Rhee and V. S. Pande, Ann. Rev. Biophys. Biomol. Struct. 34, 43 (2004).

[27] H. M. Taylor and S. Karlin, An introduction to stochastic modelling, Academic Press, Orlando (1984).

[28] Z. Bai, J. Demmel, J. Dongarra, A. Ruhe and H. van der Vorst (eds.), Templates for the Solution of Algebraic Eigenvalue Problems: a Practical Guide, SIAM, Philadelphia (2000).

[29] K. J. Laidler, Chemical Kinetics, Harper \& Row, New York (1987).

[30] O. M. Becker and M. Karplus, J. Chem. Phys. 106, 1495 (1997).

[31] D. J. Wales, M. A. Miller and T. R. Walsh, Nature 394, 758 (1998).

[32] S. Goldberg, Probability: An Introduction, Dover Publications, New York (1960).

[33] M. A. Novotny, Phys. Rev. Lett. 74, 1 (1995).

[34] S. A. Trygubenko and D. J. Wales, in preparation (2005). 
[35] J. E. Jones and A. E. Ingham, Proc. R. Soc. A 107, 636 (1925).

[36] A. L. Mackay, Acta Cryst. 15, 916 (1962).

[37] R. S. Berry, T. L. Beck, H. L. Davis and J. Jellinek, Adv. Chem. Phys. 70B, 75 (1988).

[38] P. Labastie and R. L. Whetten, Phys. Rev. Lett. 65, 1567 (1990).

[39] D. Chandler, J. Chem. Phys. 68, 2959 (1978).

[40] C. Dellago, P. G. Bolhuis and D. Chandler, J. Chem. Phys. 110, 6617 (1999). 
TABLE 1: Results for $\mathrm{LJ}_{55}$ at $k_{B} T / \epsilon=0.4$ for $t_{\mathrm{b}}$ waiting times averaged over $1000 \mathrm{KMC}$ trajectories and a fractional convergence criterion of $10^{-3}$ in the committor probability calculation. CPU times (seconds) are given in brackets for an UltraSparcIII $900 \mathrm{MHz}$ processor. The two timings in the $k_{\mathrm{AB}}^{\mathrm{NSS}}$ column refer to runs with/without leapfrog moves. For comparison, $k_{\mathrm{AB}}^{\mathrm{KMC}}=0.48 \times 10^{-7}$ averaged over 1000 trajectories, and this calculation required about 4800 s on an UltraSparcIII $900 \mathrm{MHz}$ processor both with and without leapfrog KMC moves. All rate constants are in reduced units of $\nu_{\mathrm{LJ}}$.

\begin{tabular}{ccccc}
\hline \hline$E_{\mathrm{th}} / \epsilon$ & $k_{\mathrm{AB}}^{\mathrm{SS}} \times 10^{7}$ & $k_{\mathrm{AB}}^{\mathrm{NSS}} \times 10^{7}$ & $k_{\mathrm{AB}}^{\mathrm{NSS}} / k_{\mathrm{AB}}^{\mathrm{SS}}$ & $k_{\mathrm{AB}}^{\mathrm{GT}} \times 10^{7}$ \\
\hline-266 & $1.02(1)$ & $0.50(8 / 10)$ & 0.50 & $0.50(7)$ \\
-267 & $0.46(1)$ & $0.41(7 / 9)$ & 0.89 & $0.34(8)$ \\
-268 & $0.46(1)$ & $0.41(6 / 6)$ & 0.90 & $0.34(9)$ \\
-269 & $0.46(1)$ & $0.45(5 / 5)$ & 0.96 & $0.38(8)$ \\
-270 & $0.46(1)$ & $0.44(4 / 5)$ & 0.95 & $0.38(9)$ \\
-271 & $0.53(1)$ & $0.16(11 / 8)$ & 0.29 & $0.14(22)$ \\
-272 & $0.54(3)$ & $0.15(6 / 5)$ & 0.28 & $0.15(19)$ \\
-273 & $0.58(3)$ & $0.16(5 / 5)$ & 0.27 & $0.15(22)$ \\
-274 & $1.20(3)$ & $0.23(4 / 4)$ & 0.19 & $0.24(25)$ \\
-275 & $1.20(3)$ & $0.23(4 / 4)$ & 0.19 & $0.24(25)$ \\
$\leq-276$ & $2.27(3)$ & $0.42(4 / 3)$ & 0.18 & $0.42(24)$ \\
\hline \hline
\end{tabular}


TABLE 2: Results for $\mathrm{LJ}_{55}$ at $k_{B} T / \epsilon=0.3$ for $t_{\mathrm{b}}$ waiting times averaged over $1000 \mathrm{KMC}$ trajectories, and fractional convergence criteria in the committor probability calculation of $10^{-3}$ for $E_{\mathrm{th}} \geq-270 \epsilon$ and $10^{-7}$ for $E_{\mathrm{th}} \leq-271 \epsilon$. CPU times (seconds) are given in brackets for an UltraSparcIII $900 \mathrm{MHz}$ processor. The two timings in the $k_{\mathrm{AB}}^{\mathrm{NSS}}$ column refer to runs with/without leapfrog moves. For comparison, $k_{\mathrm{AB}}^{\mathrm{KMC}}=0.13 \times 10^{-11}$ averaged over 1000 trajectories, and this calculation required about $2.0 \times 10^{7} \mathrm{~s}$ and $2.6 \times 10^{7} \mathrm{~s}$ on an UltraSparcIII $900 \mathrm{MHz}$ processor for KMC runs with and without leapfrog KMC moves, respectively. All rate constants are in reduced units of $\nu_{\mathrm{LJ}}$.

\begin{tabular}{lllll}
\hline \hline$E_{\mathrm{th}} / \epsilon$ & $k_{\mathrm{AB}}^{\mathrm{SS}} \times 10^{11}$ & $k_{\mathrm{AB}}^{\mathrm{NSS}} \times 10^{11}$ & $k_{\mathrm{AB}}^{\mathrm{NSS}} / k_{\mathrm{AB}}^{\mathrm{SS}}$ & $k_{\mathrm{AB}}^{\mathrm{GT}} \times 10^{11}$ \\
\hline-266 & $0.19(2)$ & $0.16(3 / 4)$ & 0.85 & $0.16(7)$ \\
-267 & $0.13(1)$ & $0.12(2 / 2)$ & 0.96 & $0.10(8)$ \\
-268 & $0.13(1)$ & $0.12(2 / 2)$ & 0.96 & $0.10(9)$ \\
-269 & $0.12(1)$ & $0.12(1 / 2)$ & 1.00 & $0.11(8)$ \\
-270 & $0.12(1)$ & $0.12(1 / 2)$ & 0.99 & $0.10(9)$ \\
-271 & $0.13(122)$ & $0.11(125 / 135)$ & 0.84 & $0.038(22)$ \\
-272 & $0.13(128)$ & $0.11(126 / 129)$ & 0.83 & $0.038(19)$ \\
-273 & $0.13(128)$ & $0.12(129 / 129)$ & 0.87 & $0.040(22)$ \\
-274 & $0.16(146)$ & $0.10(147 / 147)$ & 0.61 & $0.067(25)$ \\
-275 & $0.16(147)$ & $0.10(147 / 147)$ & 0.61 & $0.067(25)$ \\
$\leq-276$ & $0.17(152)$ & $0.13(152 / 152)$ & 0.77 & $0.10(24)$ \\
\hline \hline
\end{tabular}


TABLE 3: Results for $\mathrm{LJ}_{55}$ at $k_{B} T / \epsilon=0.2$ for $t_{\mathrm{b}}$ waiting times averaged over $1000 \mathrm{KMC}$ trajectories, and fractional convergence criteria in the committor probability calculation of $10^{-3}$ for $E_{\mathrm{th}} \geq-268 \epsilon, 10^{-4}$ for $-270 \epsilon \leq E_{\mathrm{th}} \leq-269 \epsilon$, and $10^{-9}$ for $E_{\mathrm{th}}=-271 \epsilon$. The $k_{\mathrm{AB}}^{\mathrm{SS}}$ result for $E_{\mathrm{th}}=-271 \epsilon$ is probably not converged even for this tighter convergence criterion, and should be considered a lower bound for comparison with $k_{\mathrm{AB}}^{\mathrm{GT}}$. CPU times (seconds) are given in brackets for an UltraSparcIII $900 \mathrm{MHz}$ processor. The two timings in the $k_{\mathrm{AB}}^{\mathrm{NSS}}$ column refer to runs with/without leapfrog moves. Conventional KMC calculations were not feasible at this temperature. All rate constants are in reduced units of $\nu_{\mathrm{LJ}}$.

\begin{tabular}{|c|c|c|c|c|}
\hline$E_{\mathrm{th}} / \epsilon$ & $k_{\mathrm{AB}}^{\mathrm{SS}} \times 10^{22}$ & $k_{\mathrm{AB}}^{\mathrm{NSS}} \times 10^{22}$ & $k_{\mathrm{AB}}^{\mathrm{NSS}} / k_{\mathrm{AB}}^{\mathrm{SS}}$ & $k_{\mathrm{AB}}^{\mathrm{GT}} \times 10^{22}$ \\
\hline-266 & $0.79(2)$ & $0.74(2 / 635)$ & 0.93 & $0.75(9)$ \\
\hline-267 & $0.65(1)$ & $0.65(2 / 36)$ & 1.00 & $0.65(10)$ \\
\hline-268 & $0.65(1)$ & $0.65(2 / 28)$ & 1.00 & $0.65(10)$ \\
\hline-269 & $0.65(1)$ & $0.65(2 / 219)$ & 1.00 & $0.65(10)$ \\
\hline-270 & $0.65(1)$ & $0.65(2 / 206)$ & 1.00 & $0.65(10)$ \\
\hline-271 & $0.092(119331)$ & - & - & $0.021(26)$ \\
\hline-272 & - & - & - & $0.022(23)$ \\
\hline-273 & - & - & - & $0.023(27)$ \\
\hline-274 & - & - & - & $0.044(31)$ \\
\hline-275 & - & - & - & $0.045(31)$ \\
\hline$\leq-276$ & - & - & - & $0.60(29)$ \\
\hline
\end{tabular}


TABLE 4: Results for $\mathrm{LJ}_{55}$ at $k_{B} T / \epsilon=0.1$ for $t_{\mathrm{b}}$ waiting times averaged over $1000 \mathrm{KMC}$ trajectories, and a fractional convergence criterion in the committor probability calculation of $10^{-3}$ for $E_{\mathrm{th}} \geq-270 \epsilon$. For thresholds of $E_{\mathrm{th}} \leq-271 \epsilon$ it proved impossible to converge the committor probability calculation for the fractional convergence criteria required to yield meaningful results. CPU times (seconds) are given in brackets for an UltraSparcIII $900 \mathrm{MHz}$ processor. The timings in the $k_{\mathrm{AB}}^{\mathrm{NSS}}$ column refer to runs with leapfrog moves; the corresponding runs without leapfrog moves were not feasible. Conventional KMC calculations were also unfeasible at this temperature. All rate constants are in reduced units of $\nu_{\mathrm{LJ}}$.

\begin{tabular}{cllcl}
\hline \hline$E_{\mathrm{th}} / \epsilon$ & $k_{\mathrm{AB}}^{\mathrm{SS}} \times 10^{52}$ & $k_{\mathrm{AB}}^{\mathrm{NSS}} \times 10^{52}$ & $k_{\mathrm{AB}}^{\mathrm{NSS}} / k_{\mathrm{AB}}^{\mathrm{SS}}$ & $k_{\mathrm{AB}}^{\mathrm{GT}} \times 10^{52}$ \\
\hline-266 & $0.14(2)$ & $0.12(3)$ & 0.87 & $0.12(8)$ \\
-267 & $0.14(2)$ & $0.14(544)$ & 1.00 & $0.14(9)$ \\
-268 & $0.14(2)$ & $0.14(3)$ & 1.00 & $0.14(11)$ \\
-269 & $0.14(3)$ & $0.14(3)$ & 1.00 & $0.14(10)$ \\
-270 & $0.14(3)$ & $0.14(20)$ & 1.00 & $0.14(10)$ \\
-271 & - & - & - & $0.012(26)$ \\
-272 & - & - & - & $0.012(23)$ \\
-273 & - & - & - & $0.013(25)$ \\
-274 & - & - & - & - \\
-275 & - & - & - & $0.075(25)$ \\
$\leq-276$ & - & - & - & $0.075(25)$ \\
\hline \hline
\end{tabular}




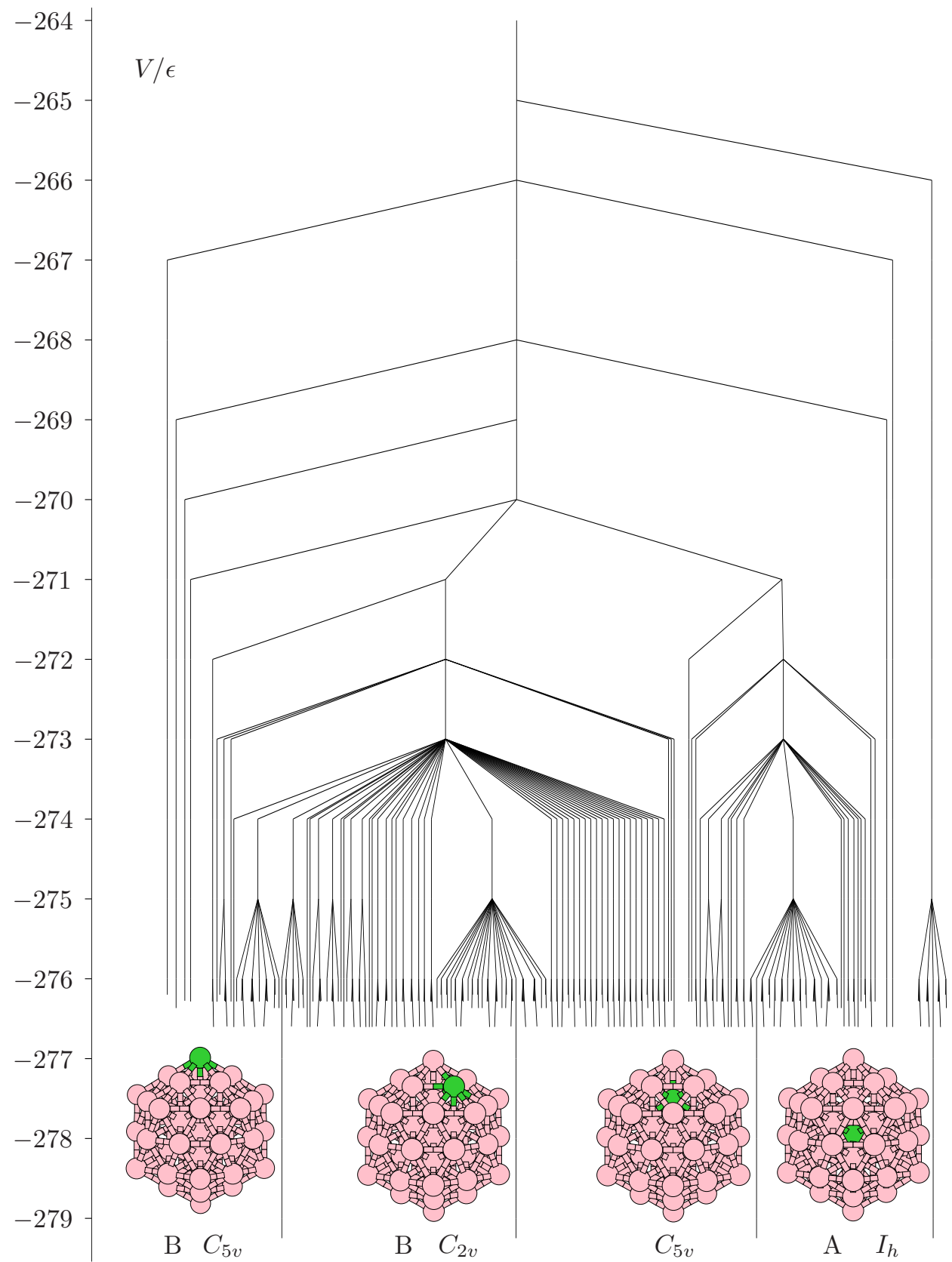

Figure 1: Disconnectivity graph for $\mathrm{LJ}_{55}$ in which permutation-inversion isomers of the shaded atom are distinguished. Permutation-inversion isomers of the other atoms are grouped together for every minimum and transition state. The tagged atom can occupy four distinct sites in the icosahedral global minimum, producing four separate branches, which are labelled according to the appropriate point group symmetry and A/B region (before any reclassification). The DPS stationary point database contains 5,609 minima and 10,134 transition states, ${ }^{9}$ but the graph includes only the lowest 250 minima for clarity. The potential energy, $V$, is in units of $\epsilon$. 